\title{
Efeito do exercício físico sobre o lipidograma, força muscular e índice antropométrico de pacientes com síndrome metabólica: Uma revisão sistemática
}

\author{
Effect of physical exercise on lipidogram, muscle strength and anthropometric index of patients \\ with metabolic syndrome: A systematic review \\ Efecto del ejercicio físico sobre lipidograma, fuerza muscular e índice antropométrico de pacientes \\ con síndrome metabólico: Una revisión sistemática
}

\author{
Arthur Mineli Kuester Berto \\ ORCID: https://orcid.org/0000-0003-0659-0219 \\ Universidade Católica de Brasília, Brasil \\ E-mail: arthurmkb7@gmail.com \\ Ludmila Rosa Faria \\ ORCID: https://orcid.org/0000-0002-3763-3966 \\ Universidade Católica de Brasília, Brasil \\ E-mail: ludvhf@gmail.com \\ Lizandra Karoline Silva do Monte \\ ORCID: https://orcid.org/0000-0001-8580-3803 \\ Universidade Católica de Brasília, Brasil \\ E-mail: lizandraksm@hotmail.com \\ Mariana Barretto Pavani \\ ORCID: https://orcid.org/0000-0003-3425-9531 \\ Universidade Católica de Brasília, Brasil \\ E-mail: marianapavoni@hotmail.com \\ Rebeka Viana Borsoi \\ ORCID: https://orcid.org/0000-0002-7800-9107 \\ Universidade Católica de Brasília, Brasil \\ E-mail: rebekaborsoi@gmail.com \\ Washington Luiz Mariano da Silva \\ ORCID: https://orcid.org/0000-0002-4214-3662 \\ Universidade Católica de Brasília, Brasil \\ E-mail: wasedufisica@gmail.com \\ Geiziane Leite Rodrigues Melo \\ ORCID: https://orcid.org/0000-0002-7800-2212 \\ Universidade Católica de Brasília, Brasil \\ E-mail: geizianemelo93@gmail.com \\ Rodrigo Vanderson Passos Neves \\ ORCID: https://orcid.org/0000-0002-3257-7870 \\ Universidade Católica de Brasília, Brasil \\ E-mail: rpassosneves@yahoo.com.br \\ Thiago Santos Rosa \\ ORCID: https://orcid.org/0000-0003-0418-0945 \\ Universidade Católica de Brasília, Brasil \\ E-mail: thiagoacsdkp@yahoo.com.br \\ Milton Rocha Moraes \\ ORCID: https://orcid.org/0000-0003-4818-9650 \\ Universidade Católica de Brasília, Brasil \\ E-mail: mrmoraes70@gmail.com
}

\begin{abstract}
Resumo
Objetivo: Determinar se a realização de exercícios físicos agudos e crônicos produz alterações significativas nos componentes metabólicos e cardiovasculares, sobretudo no perfil lipídico de indivíduos com diagnóstico de síndrome metabólica (SM). Métodos: Busca realizada em periódicos indexados nas bases de dados eletrônicas MEDLINE/PUBMED, LILACS e SCIELO sobre os efeitos dos exercícios isométricos em indivíduos com SM. Os critérios de inclusão dos estudos analisados foram: estudos publicados entre 2010 e 2020; estudos publicados em inglês e português (sendo a última em revistas indexadas); amostra de indivíduos com idade superior a 40 anos; presença de alteração do perfil lipídico dos participantes; avaliação do perfil lipídico por aparelhos de mesa e/ou portáteis e artigos originais. Resultados: Dentre os estudos encontrados nessa revisão, 12 artigos contemplaram aos
\end{abstract}


critérios propostos. Os exercícios aeróbicos foram mais recorrentes presente em 5 estudos, seguido pelo exercício combinado com aeróbico e treinamento de força totalizando 4 artigos, além de mostrarem diminuição nos parâmetros relacionados a SM, especialmente no lipidograma. Ademais, evidências sugerem que a associação entre uma dieta com restrição calórica e exercícios combinados são ferramentas primordiais para redução dos parâmetros da SM. Conclusão: Evidências sugerem que o exercício físico exerce diversos efeitos positivos sistêmicos ao indivíduo com síndrome metabólica; principalmente idosos por meio do fortalecimento muscular, coordenação corporal, redução de quedas, otimização do desempenho cardiorrespiratório, entre outras.

Palavras-chave: Síndrome metabólica; Exercício físico; Restrição calórica.

\begin{abstract}
Objective: To determine whether the performance of acute and chronic physical exercises produces significant changes in the metabolic and cardiovascular components, especially in the lipid profile of individuals diagnosed with metabolic syndrome (MS). Methods: Search conducted in journals indexed in the electronic databases MEDLINE / PUBMED, LILACS and SCIELO about the effects of isometric exercises in individuals with MS. The inclusion criteria for the studies analyzed were: studies published between 2010 and 2020; studies published in English and Portuguese (the last being in indexed journals); sample of individuals over 40 years of age; presence of alteration in the participants' lipid profile; assessment of the lipid profile by table and / or portable devices and original articles. Results: Among the studies found in this review, 12 articles met the proposed criteria. Aerobic exercises were the most recurrent present in 5 studies, followed by combined exercise with aerobic and strength training totaling 4 articles, in addition to showing a decrease in parameters related to MS, especially in the lipidogram. Furthermore, evidence suggests that the association between a calorie-restricted diet and combined exercises are essential tools for reducing the parameters of MS. Conclusion: Evidence suggests that physical exercise has several positive systemic effects on individuals with metabolic syndrome; mainly elderly people through muscle strengthening, body coordination, reduction of falls, optimization of cardiorespiratory performance, among others.
\end{abstract}

Keywords: Metabolic syndrome; Physical exercise; Caloric restriction.

\title{
Resumen
}

Objetivo: Determinar si la realización de ejercicios físicos agudos y crónicos produce cambios significativos en los componentes metabólicos y cardiovasculares, especialmente en el perfil lipídico de individuos diagnosticados con síndrome metabólico (SM). Métodos: Búsqueda realizada en revistas indexadas en las bases de datos electrónicas MEDLINE / PUBMED, LILACS y SCIELO sobre los efectos de los ejercicios isométricos en personas con EM. Los criterios de inclusión de los estudios analizados fueron: estudios publicados entre 2010 y 2020; estudios publicados en inglés y portugués (el último en revistas indexadas); muestra de individuos mayores de 40 años; presencia de alteración en el perfil lipídico de los participantes; evaluación del perfil lipídico mediante mesa y / o dispositivos portátiles y artículos originales. Resultados: Entre los estudios encontrados en esta revisión, 12 artículos cumplieron con los criterios propuestos. Los ejercicios aeróbicos fueron más frecuentes en 5 estudios, seguidos del ejercicio combinado con entrenamiento aeróbico y de fuerza totalizando 4 artículos, además de mostrar una disminución de los parámetros relacionados con la EM, especialmente en el lipidograma. Además, la evidencia sugiere que la asociación entre una dieta restringida en calorías y ejercicios combinados son herramientas esenciales para reducir los parámetros de la EM. Conclusión: La evidencia sugiere que el ejercicio físico tiene varios efectos sistémicos positivos en individuos con síndrome metabólico; principalmente personas mayores a través del fortalecimiento muscular, coordinación corporal, reducción de caídas, optimización del rendimiento cardiorrespiratorio, entre otros.

Palabras clave: Síndrome metabólico; Ejercicio físico; Restricción calórica.

\section{Introdução}

O aumento da expectativa de vida e a queda da taxa de natalidade contribuem para o envelhecimento da população brasileira, de modo que em 2012 havia 4,8 milhões de idosos enquanto que em 2017 superou a marca de 30,2 milhões, segundo a pesquisa nacional por amostra de domicílios contínua - características dos moradores e domicílios, divulgada pelo Instituto Brasileiro de Geografia e Estatística. Com o processo de envelhecimento, existe um risco maior para a síndrome metabólica (SM), devido à tendência de aumento da prevalência dos componentes da SM, como hipertensão arterial, diabetes mellitus, obesidade e dislipidemia (Saad et al., 2014).

A síndrome metabólica, caracterizada por obesidade central, dislipidemia, hiperglicemia e pressão arterial limítrofe, hoje, é um grande desafio para a saúde pública em todo o mundo, por associar-se com um importante risco para doença cardiovascular e diabetes tipo 2 (Saad et al., 2014; McCracken et al., 2018). Os idosos são o grupo da população com maior 
prevalência de eventos cardiovasculares, logo, identificar a prevalência de SM nesse público é de suma importância para medidas de controle de risco. Ademais, existe um maior risco para déficits cognitivos entre os pacientes com SM, em especial quando a aumento dos valores de glicemia (Rigo et al., 2009). Por outro lado, os idosos estão apresentando o quadro de dinapenia, com a perda de massa e força muscular, é umas das principais doenças hipocinéticas que acomete os idosos pela inatividade física pode ser um fator independente que afeta à incidência de SM (Volpe, Sukumar, Milliron, 2016; Leahy et al., 2018).

Estudos epidemiológicos e de coorte têm demonstrado forte associação entre obesidade e inatividade física, assim como tem sido relatada associação inversa entre atividade física, índice de massa corpórea, razão cintura-quadril e circunferência abdominal (Agner et al., 2018; Morales-Palomo et al., 2019). Esses estudos demonstraram os benefícios do exercício sobre a obesidade que podem ser alcançados com intensidade baixa, moderada ou alta, indicando que a manutenção de um estilo de vida ativo, independente de qual atividade praticada, pode evitar o desenvolvimento dessa doença (Agner et al., 2018; Morales-Palomo et al., 2018).

Para o tratamento da obesidade é necessário que o gasto energético seja maior que o consumo energético diário, o que nos faz pensar que uma simples redução na quantidade de comida por meio da dieta alimentar seja suficiente. No entanto, isso não é tão simples; tem sido demonstrado que mudança no estilo de vida, por intermédio aumento na quantidade de exercício físico praticado associado a reeducação alimentar, é o melhor tratamento (Delgado-Floody et al., 2019).

Embora a maioria dos estudos tenha examinado o efeito do exercício aeróbio sobre a perda de peso, a utilização do exercício de força dinâmico tem demonstrado algumas vantagens (Bateman et al., 2011; Delgado-Floody et al., 2019). O treinamento de força é um potente estímulo para aumentar a massa, força e potência muscular, podendo ajudar a preservar a musculatura, sobretudo de idosos, que tende a diminuir com o processo de envelhecimento (Moraes et al., 2012; Normandin et al., 2019).

Os efeitos do exercício aeróbio sobre o perfil de lipídios e lipoproteínas são bem conhecidos (Romero et al., 2011; Matsuo et al., 2015). Indivíduos ativos fisicamente apresentam maiores níveis de HDL colesterol e menores níveis de triglicérides, LDL e VDL colesterol, comparados a indivíduos sedentários (Chung et al., 2017). Estudos de intervenção demonstram que perfis desfavoráveis de lipídios e lipoproteínas melhoram com o treinamento físico (Chang et al., 2016; Verrusio et al., 2016). O exercício físico tem demonstrado ser eficiente em diminuir o nível de VLDL colesterol em indivíduos com diabetes do tipo 2; entretanto, com algumas exceções, a maioria dos estudos não tem demonstrado significante melhora nos níveis de HDL e LDL colesterol nessa população, talvez devido à baixa intensidade de exercício utilizada (Weatherwax, et al., 2018).

Apesar de estudos acerca do efeito do exercício físico sobre o perfil de lipídios e lipoproteínas em indivíduos com síndrome metabólica serem escassos, considerando as evidências acima e o fato de que o exercício amplia a habilidade do tecido muscular de consumir ácidos graxos e aumenta a atividade da enzima lípase lipoprotéica no músculo (Verrusio et al., 2016; Weatherwax, et al., 2018), é provável que o exercício físico seja eficiente em melhorar o perfil de lipídios e lipoproteínas em indivíduos com síndrome metabólica. No entanto, há poucos estudos relacionados às mudanças no perfil lipídico em resposta ao exercício de força isométrica.

Entretanto, a redução da massa corporal total (tecido adiposo) e do perímetro abdominal, quando necessária, adoção de um plano alimentar saudável e sustentável, redução do consumo de sódio, moderação no consumo de álcool, parar de fumar, controle do estresse e a prática regular de exercício físico são fortemente recomendadas para a prevenção e o tratamento da síndrome metabólica. Em especial, o exercício pode reduzir a morbimortalidade cardiovascular, pois há evidências em que pessoas ativas apresentam um risco $30 \%$ menor de desenvolver hipertensão arterial sistêmica (HAS) que os sedentários (Fagard, 2005). 
Portanto, com esta lacuna na literatura científica, o objetivo desta revisão foi determinar se a realização de exercícios físicos agudos e crônicos produz alterações significativas nos componentes metabólicos, cardiovasculares e na força muscular, sobretudo no perfil lipídico de indivíduos com diagnóstico de síndrome metabólica.

\section{Metodologia}

A busca foi realizada em periódicos indexados nas bases de dados eletrônicas MEDLINE/PUBMED, LILACS e SCIELO sobre os efeitos dos exercícios isométricos em indivíduos com SM. Os descritores utilizados para a busca eletrônica nas bases de dados foram identificados mediante consulta aos Descritores em Ciências de Saúde (DeCS), por meio do portal da Biblioteca Virtual em Saúde (BVS) sendo eles: síndrome metabólica e idosos e exercícios isométricos, combinados com operador "and" tanto na língua inglesa, espanhola e portuguesa. Os critérios de inclusão dos estudos analisados foram: estudos publicados entre 2010 e 2020; estudos publicados em inglês e português (sendo a última em revistas indexadas); amostra de indivíduos com idade superior a 40 anos; presença de alteração do perfil lipídico dos participantes; avaliação do perfil lipídico por aparelhos de mesa e/ou portáteis e artigos originais.

A Figura 1 apresenta o fluxograma que contém as etapas do processo de busca e seleção dos artigos incluídos na presente revisão. A partir da busca em bases de dados por meio dos descritores foram identificados, inicialmente, 2.277 artigos sendo que após a leitura do título e resumos apenas 14 artigos foram avaliados completamente para enquadramento nos critérios de inclusão. Após essa etapa 12 artigos foram incluídos na amostra da revisão.

Figura 1 - Fluxograma do levantamento bibliográfico sobre o tema síndrome metabólica, idosos e exercício físico.

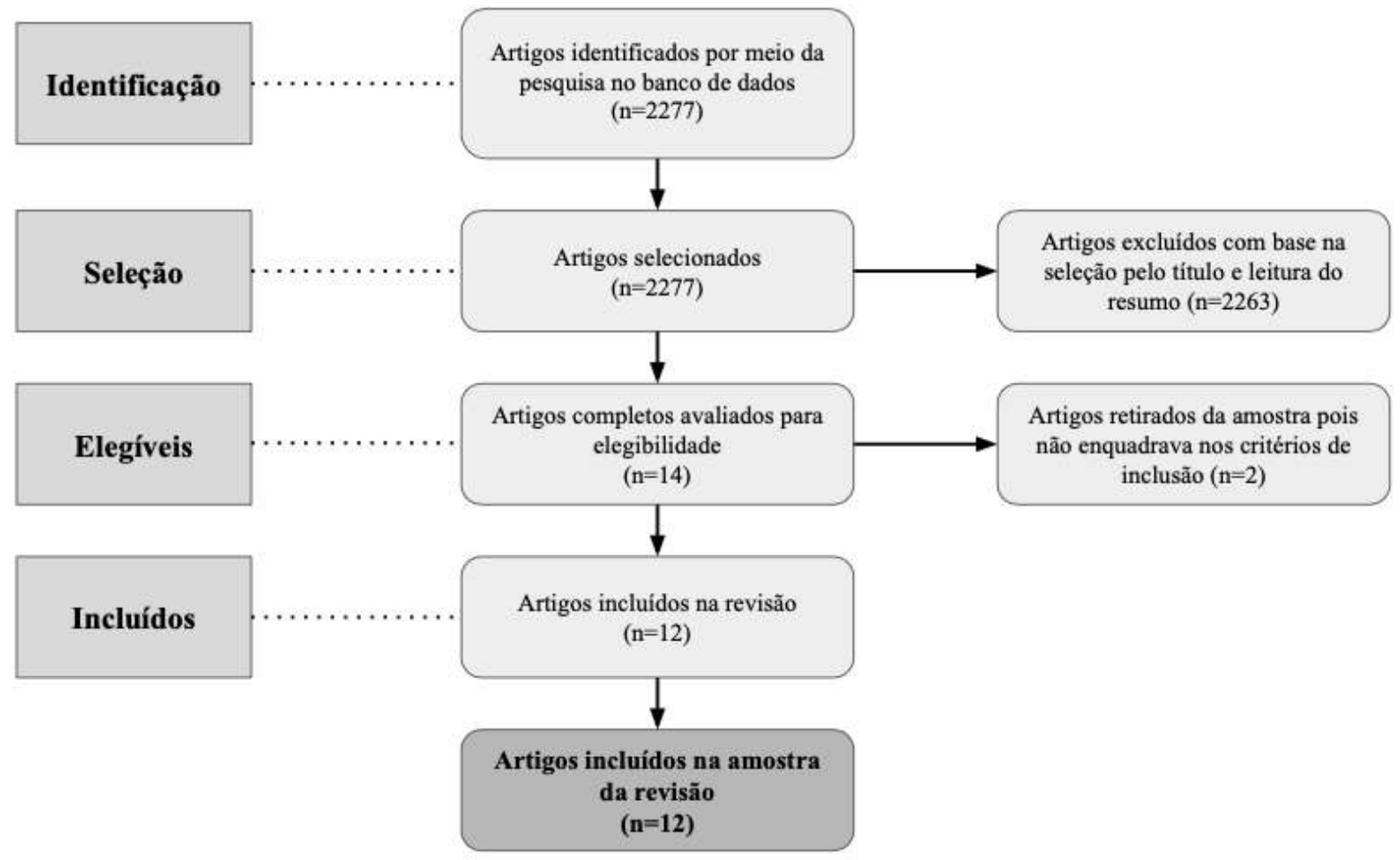

Fonte: Autores. 


\section{Resultados}

Observou que os países que mais publicaram sobre a temática da síndrome metabólica e exercício físico em adultos com superior a 40 anos foram a Espanha e o Estados Unidos da América, três estudos em ambos. A faixa etária variou entre 18 a 79 anos de idade, com amostras entre 16 a 136 indivíduos totalizando 740 participantes. A Tabela 1 descreve o efeito do exercício sobre o lipidograma, força muscular e indicadores antropométricos de pacientes com SM. Em relação ao tipo de exercício foi verificado que cinco estudos utilizaram exercício aeróbicos, sendo subdividos em Treinamento Intervalado de Alta Intensidade (HIIT) e Treinamento Contínuo de Moderada Intensidade (MICT) (Tabela1).

Já quanto ao treinamento de força está presente em três estudos, sendo apenas dinâmico; além de quatro estudos utilizarem técnicas combinadas de ambas atividades (Tabela1). Ademais, a quantidade de estudos aliando a dieta calórica restritiva as práticas foram iguais a três. Um aspecto relevante a destacar é que estudos foram compostos por uma amostra diversas com idosos, obesos mórbidos e sedentários (Tabela1). Em relação ao uso exclusivo ou combinado de exercícios isométricos não foram encontrados estudos e dados elucidando a associação desses com os parâmetros da síndrome metabólica mesmo já sabendo dos benefícios que esse tipo de exercício traz do ponto de vista cardiometabólico 
Research, Society and Development, v. 10, n. 5, e1210514623, 2021

(CC BY 4.0) | ISSN 2525-3409 | DOI: http://dx.doi.org/10.33448/rsd-v10i5.14623

Tabela 1. Características gerais dos estudos avaliados sobre síndrome metabólica e exercício físico.

\begin{tabular}{|c|c|c|c|c|c|c|}
\hline $\begin{array}{l}\text { Autor (ano) } \\
\text { País }\end{array}$ & $\begin{array}{l}\text { Características } \\
\text { dos participantes }\end{array}$ & $\begin{array}{l}\text { Metodologia } \\
\text { do Estudo }\end{array}$ & $\begin{array}{l}\text { Duração } \\
\text { Semanas }\end{array}$ & $\begin{array}{c}\text { Tipo } \\
\text { de } \\
\text { Exercício }\end{array}$ & $\begin{array}{c}\text { Análises } \\
\text { Metabólicas }\end{array}$ & Resultados \\
\hline $\begin{array}{l}\text { Morales- } \\
\text { Palomo et } \\
\text { al. } \\
(2019) \\
\text { Espanha }\end{array}$ & $\begin{array}{c}\begin{array}{c}\text { Total de } 121 \text { idosos }+ \text { MetS }+ \\
\text { Sedentários }(42 \text { mulheres e } 79 \\
\text { homens) } \\
\text { 4HIIT: } \mathrm{N}=32(55 \pm 8) \text { anos } \\
\text { MICT: } \mathrm{N}=35(57 \pm 7) \text { anos } \\
\text { 1HITT: } \mathrm{N}=32(58 \pm 8) \text { anos } \\
\text { CONTROLE: } \mathrm{N}=22(57 \pm 9) \text { anos }\end{array}\end{array}$ & 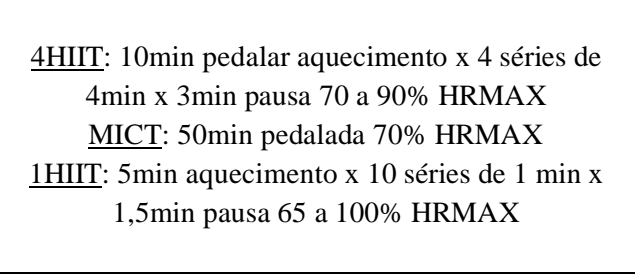 & $\begin{array}{c}\text { Duração } 16 \\
\text { semanas }\end{array}$ & AT & $\begin{array}{l}\text { Mindray BS400 } \\
\text { Chemistry } \\
\text { Analyzer (Mindray } \\
\text { Medical } \\
\text { Instrumentation, } \\
\text { Shenzhen, China) }\end{array}$ & $\begin{array}{c}\downarrow \text { TG no grupo MICT } \\
\downarrow \text { Circunferência abdominal nos grupos } \\
\text { 4HIIT e } 1 \text { HIIT } \\
\downarrow \text { IMC no grupo 4HIIT } \\
\text { HDL, CT e LDL sem alterações }\end{array}$ \\
\hline $\begin{array}{l}\text { Delgado- } \\
\text { Floddy et al. } \\
(2019) \\
\text { Espanha }\end{array}$ & $\begin{array}{l}\frac{\text { Obesos Classe } 3(\mathrm{MO})}{37,3 \pm 6,8 \text { anos }}: \mathrm{N}=14 \\
\frac{\text { Obesos Classe } 2(\mathrm{CO})}{47,2 \pm 15 \text { anos }}: \mathrm{N}=7\end{array}$ & $\begin{array}{c}\text { 10min aquecimento x } 5 \text { min alongamento x } 3 \text { séries } \\
\text { de } 4 \text { a } 8 \text { exercícios de } 1 \text { min x } 2 \text { min pausa } 20 \text { a } 40 \% \\
\text { HRMAX }\end{array}$ & $\begin{array}{l}3 \text { sessões semanais } \\
\text { de } 1 \mathrm{~h} \\
\text { Duração } 20 \\
\text { semanas }\end{array}$ & TR & $\begin{array}{l}\text { Baseline }{ }^{\circledR} \\
\text { Hydraulic Hand } \\
\text { Dynamometers, } \\
\quad \text { NextGen } \\
\text { Ergonomics Inc., } \\
\text { Quebec, Canada }\end{array}$ & $\begin{array}{c}\text { 个 HDL no grupo MO }(43.3 \pm 9.4 \text { vs. } \\
44.4 \pm 9.7 \mathrm{mg} / \mathrm{dL}, \mathrm{P}<0.05) \\
\downarrow \mathrm{TG} \text { em ambos os grupos: CO } \\
(148.6 \pm 45.3 \text { vs. } 132.9 \pm 44.1 \mathrm{mg} / \mathrm{dL}) \\
\text { MO }(141.9 \pm 53.6 \text { vs. } 113.1 \pm 42.5 \mathrm{mg} / \mathrm{dL}) \\
\downarrow \mathrm{IMC} \text { e circunferência abdominal em } \\
\text { ambos os grupos } \\
\text { 个 Força de preensão palmar no grupo CO } \\
\uparrow \text { Força muscular em ambos os grupos. }\end{array}$ \\
\hline $\begin{array}{l}\text { Weatherwax } \\
\text { et al. } \\
(2018) \\
\text { EUA }\end{array}$ & $\begin{array}{l}\text { Total de } 46 \text { indivíduos sedentários } \\
\text { Controle: } \mathrm{N}=8(45.6 \pm 7.9) \text { anos } \\
\frac{\text { Exercícios Padronizado: }}{(51.2 \pm 12.5) \text { anos }} \\
\frac{\text { Exercícios Individualizado: }}{(44.9 \pm 11.4) \text { anos }} \mathrm{N}=19\end{array}$ & $\begin{array}{c}\text { Métodos individualizados e padronizados para } \\
\text { prescrever a intensidade do treinamento de aptidão } \\
\text { cardiorrespiratória }\end{array}$ & $\begin{array}{l}3 \text { sessões semanais } \\
\text { de } 30 \text { minutos } \\
\text { Duração } 12 \\
\text { semanas }\end{array}$ & TR & $\begin{array}{l}\text { The Cholestech } \\
\text { LDX system (Alere } \\
\text { Inc., Waltham, } \\
\text { MA, USA) }\end{array}$ & $\begin{array}{c}\downarrow \text { Circunferência abdominal e PAS no } \\
\text { grupo padronizado } \\
\text { Sem alterações significativas em TG, HDL } \\
\text { e CT }\end{array}$ \\
\hline $\begin{array}{l}\text { Morales- } \\
\text { Palomo et } \\
\text { al. } \\
(2018) \\
\text { Espanha }\end{array}$ & $\begin{array}{c}44 \text { indivíduos } \\
\text { Obesos e Sedentários }(53 \pm 9) \text { anos } \\
\text { HIIT: } N=22(53 \pm 2 \text { anos }) \\
\text { Controle: } N=22(53 \pm 2) \text { anos }\end{array}$ & $\begin{array}{c}\underline{\text { HIT }} \\
\text { 10min pedalar aquecimento x } 4 \text { séries de } 4 \min x \\
\text { 3min pausa x } 5 \text { min de resfriamento } \\
70 \text { a } 90 \% \text { HRMAX }\end{array}$ & $\begin{array}{l}3 \text { sessões semanais } \\
\text { de } 43 \text { minutos por } \\
16 \text { semanas } \\
\text { Duração } 2 \text { anos } \\
\text { (Nov 2015 a Nov } \\
\text { 2017) }\end{array}$ & AT & $\begin{array}{l}\text { Mindray BS } 400 \\
\text { Chemistry } \\
\text { Analyzer (Mindray } \\
\text { Medical } \\
\text { Instrumentation, } \\
\text { Shenzhen, China) } \\
\end{array}$ & $\begin{array}{l}\text { 个 Circunferência Abdominal no grupo } \\
\text { controle } \\
\text { Sem alterações em relação aos valores } \\
\text { basais de TG e HD após } 12 \text { e } 24 \text { meses }\end{array}$ \\
\hline
\end{tabular}


Research, Society and Development, v. 10, n. 5, e1210514623, 2021

(CC BY 4.0) | ISSN 2525-3409 | DOI: http://dx.doi.org/10.33448/rsd-v10i5.14623

\begin{tabular}{|c|c|c|c|c|c|c|}
\hline $\begin{array}{l}\text { Agner et al. } \\
\quad(2018) \\
\text { Brasil }\end{array}$ & $\begin{array}{c}41 \text { indivíduos } \\
14 \text { homens e } 27 \text { mulheres com MetS } \\
\text { entre } 60 \text { a } 79 \text { anos } \\
\text { Intervenção: } \mathrm{N}=23(66.00 \pm 4.74) \\
\text { anos } \\
\text { Controle: } \mathrm{N}=18(67.28 \pm 4.80) \text { anos }\end{array}$ & 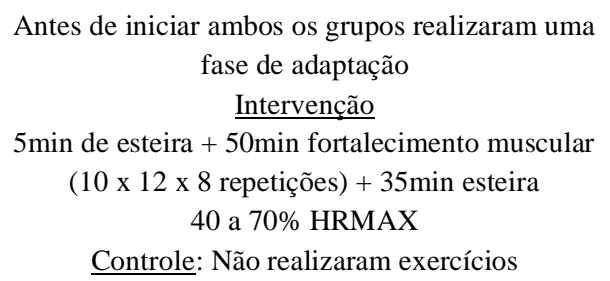 & $\begin{array}{l}2 \text { sessões por } \\
\text { semana de } 90 \\
\text { minutos durante } 12 \\
\text { semanas (total: } 24 \\
\text { sessões) }\end{array}$ & Combinado & $\begin{array}{l}\text { Accutrend Plus } \\
\text { System - } \\
\text { Diagnostics Roche }\end{array}$ & $\begin{array}{l}\text { Sem eficácia na modificação do perfil } \\
\text { metabólico dos participantes }\end{array}$ \\
\hline $\begin{array}{l}\text { Chung et al. } \\
\text { (2017) } \\
\text { Coreia do } \\
\quad \text { Sul }\end{array}$ & $\begin{array}{l}47 \text { mulheres com percentual de } \\
\text { gordura corporal } \geq 30 \%\end{array}$ & $\begin{array}{c}\text { Grupo de Sessão Única (SSG) } \\
16 \text { mulheres }(49.33 \pm 5.06) \\
1 \text { sessão de } 30 \text { minutos } \\
\text { Restrição calórica de } 300 \mathrm{kcal} \\
\text { Grupo de Sessões Múltiplas (MSG) } \\
17 \text { mulheres }(47,75 \pm 4,59 \text { anos) } \\
3 \text { sessões de } 10 \text { minutos cada com intervalo de } 4 \mathrm{~h} \\
\text { Restrição calórica de } 300 \mathrm{kcal} \\
\text { Grupo Controle }(\mathrm{CTG}) \\
14 \text { mulheres }(49.55 \pm 4.29 \text { anos) } \\
\text { Nenhum } \\
\text { Restrição calórica de } 300 \mathrm{kcal}\end{array}$ & $\begin{array}{c}3 \text { sessões por } \\
\text { semana de duração } \\
\text { de } 30 \text { min } \\
\text { Duração de } 12 \\
\text { semanas }\end{array}$ & Combinado & $\begin{array}{c}\text { Lipidograma: } \\
\text { Padrão COBAS C- } \\
111 \text { (IL, USA) } \\
9 \text { horas de jejum. } \\
\text { Amostras pré-teste } \\
\text { e pós-teste pela } \\
\text { manhã }\end{array}$ & $\begin{array}{c}\downarrow \text { Peso, IMC e massa gorda no SSG } \\
\text { 个 HDL no SSG em relação ao controle } \\
(\mathrm{p}<0,05) \\
\downarrow \text { HDL grupo controle }(10,75 \mathrm{mg} / \mathrm{dL}[95 \\
\% \mathrm{CI}=1,95-19,54], \mathrm{p}<0,01) \\
\text { 个 LDL no MSG }(-15,79 \mathrm{mg} / \mathrm{dL},[95 \% \mathrm{CI} \\
=-34,24-(-) 3,78], \mathrm{p}<0,05) \text { e CTG }(- \\
22,94 \mathrm{mg} / \mathrm{dL}[\mathrm{IC} 95 \%=-44,63-(-) 1,24], \\
\mathrm{p}<0,05)\end{array}$ \\
\hline $\begin{array}{l}\text { Normandin } \\
\text { et al. } \\
(2017) \\
\text { EUA }\end{array}$ & $\begin{array}{c}126 \text { homens e mulheres } \\
\text { Sobrepeso e Obesos } \\
\text { MetS }(69.5 \pm 3.7) \text { anos } \\
\text { Grupo de Treinamento de } \\
\text { Resistência: } \mathrm{N}=63 \\
\text { Dieta eucalórica } \\
\text { Grupo de Treinamento de Resistência } \\
\text { Dieta: } \mathrm{N}=63 \\
\text { Perda de peso moderada de } 5-10 \% \\
20220 \mathrm{kcal} / \mathrm{dia}\end{array}$ & $\begin{array}{c}3 \text { séries de } 10 \text { repetições para cada um dos } 8 \\
\text { exercícios } \\
70 \% \text { HRMAX }\end{array}$ & $\begin{array}{l}3 \text { sessões por } \\
\text { semana } \\
\text { Duração } 5 \text { meses }\end{array}$ & TR & $\begin{array}{c}\text { ND } \\
\text { Após jejum } \\
\text { noturno de } 8 \mathrm{~h}\end{array}$ & $\begin{array}{c}\downarrow \text { IMC em ambos os grupos, porém > no } \\
\text { combinado } \\
\downarrow \text { Prevalência de MetS no grupo } \\
\text { combinado } \\
\text { Sem alterações de LDL e CT } \\
\downarrow \text { VLDL }(26.2 \pm 12.2 \text { vs }-4.8 \pm 9.8 \\
\text { p=0,001) e TG }(131.0 \pm 61.2 \text { vs }-23.7 \pm \\
49.1 \mathrm{p}=0,0) \text { mais significativamente no } \\
\text { grupo combinado } \\
\text { Melhora do HDL no grupo combinado sem } \\
\text { diferença significativa entre os grupos }\end{array}$ \\
\hline $\begin{array}{l}\text { Verrusio et } \\
\text { al. } \\
(2016) \\
\text { Itália }\end{array}$ & $\begin{array}{c}30 \text { indivíduos } \\
\text { MetS } \\
50-69 \text { anos }\end{array}$ & $\begin{array}{l}\text { Grupo A: } 10 \text { indivíduos }(62.5 \pm 4.7 \text { anos) Dieta } \\
\text { Grupo B: } 10 \text { indivíduos }(60.7 \pm 6.8 \text { anos) Dieta }+ \\
\text { Exercício_75\% } \\
\text { Grupo C: } 10 \text { indivíduos (59.2 } \pm 9.1 \text { anos) Dieta + } \\
\text { Spinning } 75 \%\end{array}$ & $\begin{array}{l}\text { Grupo B: } 2 \text { sessões } \\
\text { por semana de } 1 \mathrm{~h} \\
\text { Grupo C: } 2 \text { sessões } \\
\text { por semanas de } 45 \\
\text { a } 50 \text { min } \\
\text { Duração de } 6 \\
\text { meses }\end{array}$ & Combinado & ND & $\begin{array}{l}\text { Melhora no perfil lipídico do grupo B: } \\
\downarrow \mathrm{CT}(\mathrm{df}=9 ; \mathrm{t}=5.18 ; \mathrm{p}=0.001) \\
\downarrow \mathrm{TG}(\mathrm{df}=9 ; \mathrm{t}=7.57 ; \mathrm{p}=0.001) \\
\text { Grupo } \mathrm{C} \downarrow \text { leve do colesterol total } \\
(\mathrm{df}=9 ; \mathrm{t}=2.40 ; \mathrm{p}=0.04) \\
\text { Ausência de alterações do HDL }\end{array}$ \\
\hline
\end{tabular}


Research, Society and Development, v. 10, n. 5, e1210514623, 2021

(CC BY 4.0) | ISSN 2525-3409 | DOI: http://dx.doi.org/10.33448/rsd-v10i5.14623

\begin{tabular}{|c|c|c|c|c|c|c|}
\hline $\begin{array}{l}\text { Chang et al. } \\
\text { (2016) } \\
\text { Taiwan }\end{array}$ & $\begin{array}{l}136 \text { indivíduos } \\
55,24 \pm 14,17 \text { anos } \\
\text { Obesos }+ \text { MetS } \\
\text { Grupo Controle } \\
68 \text { indivíduos } \\
\frac{\text { Grupo Intervenção }}{68 \text { indivíduos }}\end{array}$ & $\begin{array}{c}\text { Exercício aeróbico 3x semana + caminhada 5x } \\
\text { semana para ambos os grupos } \\
\text { A diferença dos grupos se deu pelo programa de } \\
\text { promoção, uma vez que o grupo intervenção eram } \\
\text { cobrados de realizar os exercícios diferente do } \\
\text { controle }\end{array}$ & $\begin{array}{l}40 \text { min } \\
\begin{array}{l}\text { Duração de } 6 \\
\text { meses }\end{array}\end{array}$ & AT & ND & $\begin{array}{c}\text { 个 HDL (2,34 mg/dL p=0,824) no grupo } \\
\text { intervenção }\end{array}$ \\
\hline $\begin{array}{l}\text { Matsuo et } \\
\text { al. } \\
(2015) \\
\text { Japão }\end{array}$ & $\begin{array}{c}26 \text { homens } 30-59 \text { anos } \\
\text { Sedentários e MetS/Pré MetS } \\
\text { HIIT: } 13 \text { homens }(47,5 \pm 7) \text { anos } \\
\text { Restrição calórica por } 4 \text { semanas } \\
\text { MICT: } 13 \text { homens }(47,4 \pm 7,5) \text { anos } \\
\text { Restrição calórica por } 4 \text { semanas }\end{array}$ & Exercícios aeróbicos assistidos & $\begin{array}{l}\text { 3x semana } \\
\text { HIIT } 18 \mathrm{~min} \\
\text { MICT } 45 \mathrm{~min} \\
\text { Duração de } 8 \\
\text { semanas }\end{array}$ & AT & $\begin{array}{l}\text { ND } \\
\text { Depois de } 12 \mathrm{~h} \text { de } \\
\text { jejum }\end{array}$ & $\uparrow$ HDL em ambos os grupos $(\mathrm{p}<0,05)$ \\
\hline $\begin{array}{l}\text { Baterman et } \\
\text { al. } \\
(2015) \\
\text { EUA }\end{array}$ & $\begin{array}{c}86 \text { indivíduos } 18 \text { a } 70 \text { anos } \\
\text { Sedentários; Sobrepeso ou obesos } \\
\text { Moderada dislipidemia } \\
\text { TR: N=31 }(51,8 \pm 11) \text { anos } \\
\text { AT: } \mathrm{N}=30(51,1 \pm 9,49) \text { anos } \\
\text { AT + RT: N=25 }(45,8 \pm 11,8) \text { anos }\end{array}$ & 3 séries de $8-\frac{\mathrm{TR}:}{12 \text { repetições/série }}$ & $\begin{array}{l}\text { 3x semana } \\
\begin{array}{l}\text { Duração de } 8 \\
\text { meses }\end{array}\end{array}$ & Combinado & $\begin{array}{l}\text { Nuclear magnetic } \\
\text { resonance } \\
\text { spectroscopy } \\
\text { (LipoScience, } \\
\text { Raleigh, North } \\
\text { Carolina) } \\
20 \text { minutos de } \\
\text { intervalo e média }\end{array}$ & $\begin{array}{c}\downarrow \text { IMC no grupo AT e AT/TR } \\
\downarrow \text { Circunferência abdominal no grupo } \\
\text { AT/TR } \\
\text { 个 Força muscular no grupo TR } \\
\downarrow \text { TG no grupo AT }(-21 \pm 56 \mathrm{p}=0,049) \text { e } \\
\text { AT/TR }(-30,1 \pm 49,8 \mathrm{p}=0,006) \\
\text { Sem alterações no HDL e CT }\end{array}$ \\
\hline $\begin{array}{l}\text { Romero et } \\
\text { al. (2014) } \\
\text { México }\end{array}$ & 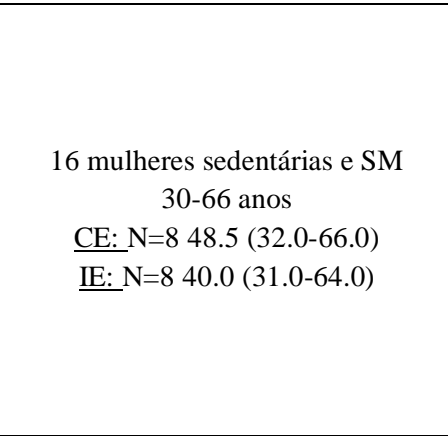 & $\begin{array}{l}\text { Exercícios aeróbicos alternando esteira e bicicleta } \\
\text { ergométrica } \\
\text { CE: } 45 \text { min de exercício aeróbico a } 65-70 \% \text { da FCR } \\
\text { IE: } 5 \text { intervalos de } 3 \text { min em alta intensidade ( } 80- \\
85 \% \text { da FCR) + recuperação ativa de } 2 \text { min }\end{array}$ & $\begin{array}{l}\text { 3x semana de } \\
\text { duração de } 55 \mathrm{~min} \\
\text { (CE) e } 35 \mathrm{~min} \text { (IE) } \\
\begin{array}{c}\text { Duração de } 16 \\
\text { semanas }\end{array}\end{array}$ & AT & $\begin{array}{c}\text { ND } \\
8 \text { horas de jejum }\end{array}$ & $\begin{array}{c}\downarrow \text { TG ambos os grupos }(\mathrm{CE}: \mathrm{Z}=-2,521 ; \mathrm{p} \\
=0,012 ; \mathrm{IE}: \mathrm{Z}=-1,96 ; \mathrm{p}=0,05) \text { atingindo } \\
\text { valores normais } \\
\downarrow \text { HDL no grupo IE }(\mathrm{Z}=-1,992 ; \mathrm{p}= \\
0,046) \\
25 \% \text { dos participantes do grupo EC não } \\
\text { tinham mais EM, em comparação com } \\
62,5 \% \text { no grupo IE } \\
\downarrow \text { IMC em ambos os grupos } \\
\downarrow \text { Massa gorda e } \uparrow \text { Força muscular no } \\
\text { grupo CE }\end{array}$ \\
\hline
\end{tabular}

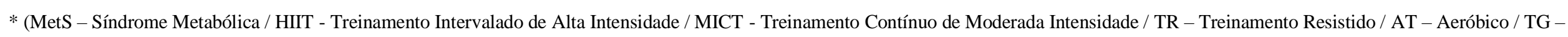

Triglicerídeos / CT - Colesterol Total / ND - Não Declarado / CE - Exercício Contínuo / IE - Exercício Intermitente / FCR - Frequência Cardiorrespiratória). Fonte: Autores 


\section{Discussão}

A cerca da análise dos estudos selecionados pode se observar que a atividade física exerce diversos efeitos positivos sistêmicos ao indivíduo com síndrome metabólica; principalmente idosos por meio do fortalecimento muscular, coordenação corporal, redução de quedas, otimização do desempenho cardiorrespiratório, entre outras (Verrusio et al, 2016). Além disso, a prática regular de atividade física ajudou em cerca de 49,2\% dos indivíduos obesos com síndrome metabólica a se tornarem metabolicamente normais após 6 meses (Chang et al, 2016).

Dessa forma, para ratificar esse panorama apresentado acima se encontra no estudo de Morales-Palomo et al (2019) que 121 idosos sedentários e com diagnóstico de SM tiveram suas medidas antropométricas e lipídicas averiguadas antes e após 16 semanas de exercícios aeróbicos de diferentes volumes e intensidades, sendo demonstrado uma redução de triglicerídeos (TG) no grupo submetido a treinamento continuo de moderada intensidade bem como redução da circunferência abdominal e IMC. Com isso, o mesmo demonstra que o principal fator de promoção de saúde nesse perfil de indivíduos é o volume aplicado. Resultados semelhantes foram verificados no estudo de Delgado-Floddy et al (2019) em que o treinamento de força de 20 semanas em pacientes com obesidade mórbida e diagnóstico de SM reduziu significativamente o TG e índice de massa corporal (IMC) de ambos os grupos, e, portanto, concluíram que maior volume de exercícios pode promover benefícios nos fatores de risco cardiometabólicos dos mesmos.

Além disso, Romero et al (2014) demonstraram que 16 mulheres sedentárias quando submetidas a exercícios contínuos e intermitentes melhoram o perfil lipídico, além de averiguar que os ambos os programas influenciaram o metabolismo lipídico e que programas de exercícios aeróbicos com menor volume e maior intensidade são eficazes para a prática regular e podem influenciar na prevenção de processos maléficos a saúde de adultas com SM.

Ademais, Verrusio et al (2016) e Baterman et al (2011) demonstram em estudos utilizando exercícios combinados de treinamento de força e aeróbico melhoram o perfil lipídico que se deu preferencialmente em exercícios aeróbicos haja vista que, essa ocorreu no âmbito da classificação e escore de SM utilizado nas pesquisas. Diante disso, é possível verificar que os estudos apresentam resultados positivos com alteração de parâmetros de SM, pois estão associados a uma dieta restritiva e prática de exercício físico os quais foram fundamentais para alterar fatores de SM tais como: ingestão dietética, comportamento sedentário, duração do sono e estresse crônico (Weatherwax et al., 2018).

Alguns estudos como os de Morales-Palomo et al (2018) e Agner et al (2018) apresentaram resultados conflitantes, pois os valores de basais do lipidograma estavam dentro do alvo terapêutico, devido a interferência do uso de medicamentos pelos participantes. Entretanto, parâmetros antropométricos como circunferência abdominal, IMC, peso e PA diminuíram com intervenção bem como apresentado nos resultados de Delgado-Floddy et al (2019) e Weatherwax et al (2018). No contexto de indivíduos com SM fica evidente a associação benéfica do exercício físico nos componentes e fatores cardiometabólicos, principalmente aqueles relacionados a força muscular.

Diante disso, é fundamental que a prescrição do exercício deve ser individualizada, afim de verificar se os indivíduos com SM estão em tratamento. Além disso, é importante que haja fomentação de ambientes com a presença de apoio social e profissionais qualificados para auxiliar na motivação a continuação ao processo, tendo vista que, há um problema na manutenção dos ganhos obtidos (diminuição do percentual de gordura e melhora dos parâmetros do lipidograma) após o término da intervenção. Com isso, formação de parques, centros de exercícios e centros comunitários são fundamentais quando associado a uma prescrição de exercício adequada e individualizada.

\section{Conclusão}

Conclui-se que o exercício aeróbico foi mais prevalente para as mudanças de parâmetros relacionados a síndrome 
metabólica especialmente do ponto de vista do lipidograma, se observa que a associação entre uma dieta com a restrição calórica e exercícios combinados são as ferramentas primordiais para a promoção da qualidade de vida dos portadores da síndrome. Entretanto, em relação aos estudos verificados há ainda um hiato importante na literatura sobre as respostas do exercício isométrico sobre a SM o que conduz e apresenta a necessidade de mais estudos sobre essa perspectiva. A produção de trabalhos com maiores amostras, maior tempo de investigação e que relatem a descrição de equipamentos utilizados para a obtenção dos dados apresentados também se faz necessária para uma maior validação estrutural e quantitativas dos demais estudos.

\section{Referências}

Agner, V. F. C., et al (2018). Effects of concurrent training on muscle strength in older adults with metabolic syndrome: a randomized controlled clinical trial.: A randomized controlled clinical trial. Archives Of Gerontology And Geriatrics, 75, 158-164. http://dx.doi.org/10.1016/j.archger.2017.12.011.

Bateman, L. A., et al (2011). Comparison of Aerobic Versus Resistance Exercise Training Effects on Metabolic Syndrome (from the Studies of a Targeted Risk Reduction Intervention Through Defined Exercise - STRRIDE-AT/RT). The American Journal Of Cardiology, 108 (6), 838-844. http://dx.doi.org/10.1016/j.amjcard.2011.04.037.

Chang, S.-H., et al (2016). Effectiveness of community-based exercise intervention programme in obese adults with metabolic syndrome. Journal Of Clinical Nursing, 25 (17-18), 2579-2589. http://dx.doi.org/10.1111/jocn.13301.

Chung, J., et al (2017). Effects of prolonged exercise versus multiple short exercise sessions on risk for metabolic syndrome and the atherogenic index in middle-aged obese women: a randomised controlled trial.: a randomised controlled trial. Bmc Women's Health, 17 (1), 1-9. http://dx.doi.org/10.1186/s12905017-0421-z.

Cornelissen, V. A., \& Fagard, R. H. Effect of resistance training on resting blood pressure: a meta-analysis of randomized controlled trials. $J$ Hypertens (2005), 23.

Delgado-Floody, P., et al (2019). Preventing metabolic syndrome in morbid obesity with resistance training: reporting interindividual variability.: Reporting interindividual variability. Nutrition, Metabolism And Cardiovascular Diseases, 29(12), 1368-1381. http://dx.doi.org/10.1016/j.numecd.2019.07.002.

Instituto Brasileiro De Geografia E Estatística (IBGE). Projeções da população: Brasil e unidades da federação: revisão 2018. Coordenação de população e indicadores sociais, 57.

Leahy, S., et al. Dynapaenic obesity and its association with health outcomes in older adult populations: protocol for a systematic review. BMJ Open $2019,9$. doi: 10.1136/bmjopen-2018-027728

Matsuo, T., So, R., Shimojo, N., \& Tanaka, K. (2015). Effect of aerobic exercise training followed by a low-calorie diet on metabolic syndrome risk factors in men. Nutrition, Metabolism And Cardiovascular Diseases, 25 (9), 832-838. http://dx.doi.org/10.1016/j.numecd.2015.05.009.

Mccracken, E, Monaghan, M, \& Sreenivasan, S. Clin Pathophysiology of the metabolic syndrome. Dermatol. (2018), 36(1):14-20. doi: 10.1016/j.clindermatol.2017.09.004.

Moraes, M. R., et al (2012). Chronic conventional resistance exercise reduces blood pressure in stage 1 hypertensive men. J Strength Cond Res, 26 (4), 1122-9. doi: 10.1519/JSC.0b013e31822dfc5e.

Morales-Palomo, F., et al (2019). Effectiveness of Aerobic Exercise Programs for Health Promotion in Metabolic Syndrome. Medicine \& Science In Sports \& Exercise, 51(9), 1876-1883. http://dx.doi.org/10.1249/mss.0000000000001983.

Morales-Palomo, F., et al (2018). Exercise Periodization over the Year Improves Metabolic Syndrome and Medication Use. Medicine \& Science In Sports \& Exercise, 50(10), 1983-1991. http://dx.doi.org/10.1249/mss.0000000000001659.

Normandin, E., et al (2017). Effect of Resistance Training and Caloric Restriction on the Metabolic Syndrome. Medicine \& Science In Sports \& Exercise, 49(3), 13-419. http://dx.doi.org/10.1249/mss.0000000000001122.

Rigo, J. C., et al. Prevalência de Síndrome Metabólica em Idosos de uma Comunidade: Comparação entre Três Métodos. Arq Bras Cardiol 2009, 93(2): 85-91 Romero, R. S., et al (2014). Effectiveness of two aerobic exercise programs in the treatment of metabolic syndrome: a preliminary study: a preliminary study. Gaceta Médica de México, 486-493.

Saad, M. A., et al (2014). Prevalence of metabolic syndrome in elderly and agreement among four diagnostic criteria. Arq Bras Cardiol. 102(3), 263-9. $10.5935 / \mathrm{abc} .20140013$.

Verrusio, W., et al (2016). Efficacy and safety of spinning exercise in middle-aged and older adults with metabolic syndrome: randomized control trial.:randomized control trial. AnnaliDell'istitutoSuperiorediSanità, 52(2), 295-300. http://dx.doi.org/10.4415/ANN_16_02_24.

Volpe, S. L., et al. Obesity Prevention in Older Adults. Curr Obes Rep. 5(2):166-75. 10.1007/s13679-016-0213-z.

Weatherwax, R. M., et al (2018). Changes in Metabolic Syndrome Severity Following Individualized Versus Standardized Exercise Prescription: a feasibility study: A Feasibility Study. International Journal Of Environmental Research And Public Health, 15(11), 2594. http://dx.doi.org/10.3390/ijerph15112594. 